\title{
DNA fragmentation factors 40 and 45 (DFF40/ DFF45) and B-cell lymphoma 2 (Bcl-2) protein are underexpressed in uterine leiomyosarcomas and may predict survival
}

\author{
This article was published in the following Dove Press journal: \\ OncoTargets and Therapy \\ 14 September 2017 \\ Number of times this article has been viewed
}

\author{
Tomasz Banas' \\ Kazimierz Pitynski' \\ Krzysztof Okon ${ }^{2}$ \\ Aleksandra Czerw ${ }^{3,4}$ \\ 'Department of Gynecology \\ and Oncology, ${ }^{2}$ Department of \\ Pathomorphology, Jagiellonian \\ University Medical College, Krakow, \\ ${ }^{3}$ Department of Public Health, \\ Faculty of Health Science, Medical \\ University of Warsaw, ${ }^{4}$ Department of \\ Health Promotion and Postgraduate \\ Education, National Institute of \\ Public Health - National Institute of \\ Hygiene, Warsaw, Poland
}

Objectives: DNA fragmentation factors 40 and 45 (DFF40 and DFF45) are responsible for final DNA-laddering during apoptosis, whereas Bcl-2 (B-cell lymphoma 2) is an apoptosis inhibitor. Our aim was to investigate the expression of DFF40, DFF45, and Bcl-2 in uterine leiomyosarcomas (uLMS), leiomyomas (uLM), and the normal myometrium. Furthermore, the correlation between DFF40, DFF45, and Bcl-2 expression and clinicopathological parameters in leiomyosarcomas was assessed. Their prognostic value in disease-free survival (DFS) and overall survival (OS) was also calculated.

Materials and methods: This study included 53 cases of uLMS from patients matched for age and menopausal status with 53 cases of uLM and 53 controls of normal myometrium (uM). Case samples of uterine myometrium from leiomyosarcomas (uLMS-M) and leiomyomas (uLM-M) were also studied. Immunohistochemical scoring was undertaken for DFF40, DFF45, and Bcl-2.

Results: DFF40, DFF45, and Bcl-2 were significantly underexpressed in uLMS compared with uLMS-M and uM. In uLMS samples, no correlation between the analyzed proteins was observed. Negative DFF40 and Bcl-2, but not DFF45, staining was a predictor of poorer DFS and OS in women with uLMS. uLM showed DFF40 and Bcl-2 overexpression compared with uM and uLM-M, with a significant positive correlation between DFF40 and DFF45. No differences in DFF40, DFF45, and Bcl-2 expression were observed between the uLMS-M, uLM-M, and uM samples, with a significant positive correlation between DFF40 and DFF45 expression.

Conclusion: DFF40, DFF45, and Bcl-2 are significantly underexpressed in uLMS, but only a lack of DFF40 and Bcl-2 negatively influences DFS and OS. Disruption of DFF40 and DFF45 expression was observed in uLMS, but not in uLM or control and case myometrium; this may play a role in tumor pathogenesis.

Keywords: B-cell lymphoma 2, disease-free survival, DNA fragmentation factor 40, DNA fragmentation factor 45, uterine, immunohistochemistry, leiomyosarcoma, uterine leiomyoma, overall survival

\section{Introduction}

Uterine leiomyosarcomas (uLMS) are rare malignant tumors arising within the myometrium, and account for $1 \%-2 \%$ of all uterine corpus malignancies; however, cervical localization of uLMS has also been reported. ${ }^{1-3}$ These represent the most common subtype of uterine sarcoma, with a stable incidence over the past three decades. ${ }^{4,5}$ Prior pelvic irradiation, use of tamoxifen, and African-American ethnicity have been reported to increase the risk of $\mathrm{uLMS}^{6-8}$
Correspondence: Tomasz Banas Department of Gynecology and Oncology, Jagiellonian University Medical College, 23 Kopernika Street, 30-50I

Krakow, Poland

$\mathrm{Tel}+48 \quad$ I 4248584

Fax +48 I2 4248560

Email tbanas@mp.pl 
In contrast to uLMS, uterine leiomyoma (uLM) is the most common gynecological pathology, affecting up to $80 \%$ of the female population. ${ }^{9}$ Although possible progression from $\mathrm{ULM}$ to uLMS has been postulated, the risk of malignant transformation of uLM is low, accounting for less than $1 \%$ of all cases. Recent clonality studies have confirmed that most uLMS arise de novo. ${ }^{10-12}$ Proven clinical and pathological prognostic factors for uLMS are age, stage, grade $(G)$, and mitotic index (MI), whereas prognostic biomarkers have not been consistently shown. ${ }^{5,13-15}$

Changes in the expression of Bcl-2, an anti-apoptotic protein, have been reported in several malignancies, including breast and cervical cancers, ovarian malignant epithelial tumors, endometrial carcinomas, as well as in uLMS; these tumors also show downregulated p53 and caspase-9 expression. ${ }^{16-22}$ Caspases (cysteine-aspartic proteases) can be activated via the mitochondrial or cytoplasmic pathways and as a result of cellular oxidative stress; their activation leads to activation of DNA fragmentation factors (DFF) in the nucleus, which are directly responsible for DNA fragmentation and cellular apoptosis. ${ }^{23}$ The DFF complex exists as a dimer composed of DFF40 (caspase-activated DNase; CAD) and DFF45 (inhibitor of caspase-activated DNase; ICAD) ${ }^{24}$ After DFF45 cleavage by caspase-3, the active form of DFF40 is released, which promotes DNA fragmentation (DNA laddering) ${ }^{24}$ However, DFF45 is not a simple inhibitor of DFF40, as its presence is essential for the proper folding of DFF40 into the active protein. ${ }^{24}$

The association between expression of these proteins and prognosis in various tumors has also been investigated. According to Hwang et al, Bcl-2 expression was a strong favorable prognostic factor in the HR(+)/HER2(-) subtype of breast cancer and a marginally significant favorable prognosticator in the HR(+)/HER2(+), but had no prognostic impact on other subtypes..$^{29}$ In contrast, no significant correlation was seen between Bcl-2 expression and clinicopathological parameters of colorectal cancer. ${ }^{26}$ Lack of Bcl-2 expression was also shown to be an independent predictor of poor prognosis in endometrial cancers. ${ }^{30,31}$ In human uterine smooth muscle tumors, a significantly lower Bcl-2 expression was observed in leiomyosarcomas than in leiomyomas, and positive Bcl-2 expression has been proven to be an independent predictor of favorable prognosis in patients with leiomyosarcomas. ${ }^{32}$ Decreased DFF45 expression has been observed in renal, colorectal, and esophageal cancers, and it has been postulated that its downregulation (although unexpected at first glance) may play a role in tumor escape from apoptosis and may promote tumor progression and metastasis. ${ }^{33-35}$
The aim of this retrospective case-control study was to evaluate the expression of DFF40, DFF45, and Bcl-2 as potential prognostic biomarkers in uLMS.

\section{Materials and methods Case selection}

In this retrospective study, 57 patients with primary uLMS were identified between 2000 and 2015. Inclusion criteria were patients older than 18 years with pathologically confirmed primary solitary uLMS; their histological diagnosis was reevaluated and confirmed. Patients who had additional malignancies, were smokers, had received hormonal treatment (including hormonal contraception) over the past 5 years, had been diagnosed with smooth muscle tumors of uncertain malignant potential or cellular and bizarre leiomyoma, or had missing clinical data were excluded. Finally, 53 uLMS cases were compared with 53 age-matched cases of solitary uLM and 53 matched cases of normal human uterine myometrium (uM). In cases of uLMS and uLM, additional myometrial samples were obtained at least $20 \mathrm{~mm}$ away from the primary tumor.

Every uLMS was staged according to the International Federation of Gynecology and Obstetrics (FIGO) 2009 classification, and the tumor grade and MI were calculated for all uLMS as the number of mitoses in 10 consecutive high-power fields (hpf) at $400 \times$ magnification that were also analyzed. Patients with stage I and II uLMS underwent total hysterectomy with bilateral salpingo-oophorectomy (BSO) and pelvic lymph node biopsy, whereas those with stages III and IV disease underwent complete cytoreductive surgery, which allowed us to obtain archive specimens of uLMS tissue and concomitant uterine myometrium. Women with uLM underwent supracervical hysterectomy with bilateral salpingectomy or salpingo-oophorectomy, if clinically justified, to obtain uLM and related uterine myometrium specimens. Additionally, uM was obtained from patients who suffered from recurrent or persistent cervical dysplasia with no otherwise diagnosed myometrial pathology, and total hysterectomy was undertaken as the final treatment. All specimens were collected premenstrually, in the first phase of the menstrual cycle, which was confirmed using histopathological examination of the concomitant endometrium. Clinical data were retrieved from medical files.

\section{Immunohistochemistry}

Detailed immunohistochemistry protocols have been presented in earlier reports. ${ }^{36,37} \mathrm{In}$ brief, $3-\mu \mathrm{m}$ sections of selected tissues were deparaffinized and rehydrated, then incubated 
in a target retrieval solution (Dako, Carpinteria, CA, USA) for $15 \mathrm{~min}$ at $98^{\circ} \mathrm{C}$. They were then incubated with $3 \%$ hydrogen peroxide in methanol for $10 \mathrm{~min}$, and washed with TRISbuffered saline ( $\mathrm{pH}$ 7.5) for a further $10 \mathrm{~min}$. The sections were incubated with diluted normal serum as a blocking solution for $30 \mathrm{~min}$. A standard immunohistochemical technique was performed using rabbit polyclonal antibodies to DFF40 (Abcam, Cambridge, UK) (1:100 dilution) and DFF45 (Abcam, Cambridge, UK) (1:50 dilution), and a monoclonal mouse anti-human antibody to Bcl-2 (1:200 dilution; Leica Microsystems GmbH, Leica Biosystems, Nussloch, Germany). Colon carcinoma (for Bcl-2), breast carcinoma (for DFF45), and ovary tissue (for DFF40) were used as positive controls. For the negative control, the same specimens and methods were used, but the primary antibodies were omitted.

\section{Immunohistochemical scoring}

DFF45, DFF40, and Bcl-2 staining was blindly and independently evaluated by two board-certified pathologists in 5 hpf of maximal staining intensity. The Remmele and Stanger immunoreactive score was used, and each sample was scored based on the intensity of staining ( 0 , no staining; 1, weak staining; 2 , moderate staining; and 3, strong staining) and the number of stained cells ( 0 , expression in up to $10 \%$ of the cells; $1+$, expression in $10 \%-50 \%$ of the cells; $2+$, expression in $51 \%-80 \%$ of the cells; and $3+$, expression in more than $80 \%$ of the cells). ${ }^{38}$ The final immunoreactivity score was determined by multiplying the intensity scores by the extent of the positivity scores of the stained cells. A discrepancy between the observations by the two pathologists occurred in $18(2.74 \%)$ cases, and the samples were verified again 2 weeks after the primary evaluation to prevent recall bias. In all cases, a consensus was achieved and no samples required exclusion from the analysis.

\section{Statistical analyses}

Clinical features presented non-normal distributions (confirmed using the Kolmogorov-Smirnov test) and are presented as the medians and interquartile range (IQR) or rough number and percentage $(\%)$. They were compared using the MannWhitney $U$ test, or the Kruskal-Wallis analysis of variance test, depending on the number of groups. Post hoc tests were used where appropriate. Immunohistochemical scoring of tissue specimens was evaluated using the chi-square test. ${ }^{39}$ Data from the immunohistochemistry results are presented as numbers and percentage of immunoscoring. Spearman's rank test was used to determine the correlation of immunoscoring and pathological features. A Kaplan-Meier survival curve was prepared according to the DFF40, DFF45, and Bcl-2 expression status. Cox's proportional hazard model was used to identify survival predictors, and the Cox-Mantel test was used to compare overall survival (OS) (defined as the period between initial surgery and the time of death) and disease-free survival (DFS) (defined as the period between initial surgery and the time of recurrence). To evaluate the intra-rater agreement of the immunohistochemistry results, the intraclass correlation coefficient (ICC) for a single histopathological evaluation with a $95 \%$ confidence interval (CI) was calculated. To randomize patients who underwent evaluation for intra-rater agreement, we used the Research Randomizer (www.randomizer.org), and 55 (20.91\%) samples were chosen and reevaluated separately for DFF40, DFF45, and Bcl-2 after a 2-week interval from the primary evaluation to prevent recall bias. The Guidelines for Reporting Reliability and Agreement in Studies were used to verify these results. ${ }^{40}$ A $p$-value of less than 0.05 was considered significant. All calculations were conducted using STATISTICA version 12.0 (StatSoft, Inc., Tulsa, OK, USA) and MedCalc Statistical Software version 17.0.4 (MedCalc Software bvba, Ostend, Belgium).

\section{Ethical approval and informed consent}

This retrospective, case-control study was approved by the Jagiellonian University Review Board (decision no: 122.6120.360) with the required informed consents from participants. All procedures were carried out in accordance with the ethical standards of the institutional and national research committees and in the spirit of the principles of the 1964 Declaration of Helsinki and its later amendments.

\section{Results \\ Clinicopathological variables}

The median age of the 53 patients with uLMS was 51 years (IQR 8). The number of cases with each FIGO stage was as follows: 27 (50.95\%) with Stage I, 5 (9.43\%) with Stage II, $12(22.64 \%)$ with Stage III, and $9(16.98 \%)$ with Stage IV. The tumor grading $(\mathrm{G})$ was as follows: $\mathrm{G}_{1} 6$ cases $(11.32 \%) ; \mathrm{G}_{2} 10$ cases $(18.87 \%)$, and $\mathrm{G}_{3} 37$ cases $(69.81 \%)$, with a median MI of 37.00 (IQR 30.00; range 12.00-123.00). The median OS was 58.00 months (IQR 54.00; range 6-155) and the median DFS was 31.00 months (IQR 34.00; range 2-155). As cases were matched according to age and menopausal status, there were no differences in the last two factors. The control group showed a significantly higher mean age of menarche compared with women with uLMS ( $p=0.002)$ and uLM ( $p=0.012$; Table 1$)$. No differences in parity and menstrual cycle characteristics 
Table I Patient characteristics for uterine leiomyosarcoma (uLMS), uterine leiomyoma (uLM), and normal myometrium cases (uM)

\begin{tabular}{|c|c|c|c|c|}
\hline & $\begin{array}{l}\text { Uterine } \\
\text { leiomyosarcomas } \\
\text { (uLMS) } n=53\end{array}$ & $\begin{array}{l}\text { Uterine } \\
\text { leiomyomas } \\
\text { (uLM) } n=53\end{array}$ & $\begin{array}{l}\text { Uterine control } \\
\text { myometrium } \\
\text { (uM) } n=53\end{array}$ & p-value \\
\hline Median age at therapy, years (IQR); min-max & & $\begin{array}{l}51.00(8.00) \\
44.00-70.00\end{array}$ & & $\mathrm{n} / \mathrm{a}$ \\
\hline $\begin{array}{l}\text { Menopausal status, } \mathrm{n}(\%) \\
\text { Pre- } \\
\text { Post }\end{array}$ & & $\begin{array}{l}30(56.61 \%) \\
23(43.39 \%)\end{array}$ & & $\mathrm{n} / \mathrm{a}$ \\
\hline $\begin{array}{l}\text { FIGO stage, } \mathrm{n}(\%) \\
\text { I } \\
\text { II } \\
\text { III } \\
\text { IV }\end{array}$ & $\begin{array}{l}27(50.95 \%) \\
5(9.43 \%) \\
12(22.64 \%) \\
9(16.98 \%)\end{array}$ & $\mathrm{n} / \mathrm{a}$ & $\mathrm{n} / \mathrm{a}$ & $\mathrm{n} / \mathrm{a}$ \\
\hline $\begin{array}{l}\text { Grade, n (\%) } \\
\qquad \begin{array}{l}1 \\
2 \\
3\end{array}\end{array}$ & $\begin{array}{l}6(11.32 \%) \\
10(18.87 \%) \\
37(68.81 \%)\end{array}$ & $\mathrm{n} / \mathrm{a}$ & $\mathrm{n} / \mathrm{a}$ & $\mathrm{n} / \mathrm{a}$ \\
\hline Median primary tumor size, cm (IQR); min-max & $\begin{array}{l}5.00(7.00) \\
2.00-15.00\end{array}$ & $\begin{array}{l}6.00(2.00) \\
3.00-13.00\end{array}$ & $\mathrm{n} / \mathrm{a}$ & 0.733 \\
\hline $\begin{array}{l}\text { Mitotic index, no of mitoses per I high power field, n (\%) } \\
\leq 50 \\
>50\end{array}$ & $\begin{array}{l}38(71.70 \%) \\
15(28.30 \%)\end{array}$ & $\mathrm{n} / \mathrm{a}$ & $\mathrm{n} / \mathrm{a}$ & $\mathrm{n} / \mathrm{a}$ \\
\hline $\begin{array}{l}\text { Adjuvant treatment, } \mathrm{n}(\%) \\
\text { None } \\
\text { Radiotherapy }(\mathrm{RTH}) \\
\text { Chemotherapy }(\mathrm{CHT}) \\
\text { Combination of } \mathrm{RTH} \text { and } \mathrm{CHT}\end{array}$ & $\begin{array}{l}21(39.63 \%) \\
5(9.43 \%) \\
22(41.51 \%) \\
5(9.43 \%)\end{array}$ & $\mathrm{n} / \mathrm{a}$ & $\mathrm{n} / \mathrm{a}$ & $\mathrm{n} / \mathrm{a}$ \\
\hline Median age of first menstrual period, years (IQR); min-max & $\begin{array}{l}12.00(2.00) \\
9.00-15.00\end{array}$ & $\begin{array}{l}12.00(2.00) \\
10.00-15.00\end{array}$ & $\begin{array}{l}13.00(1.00) \\
10.00-15.00\end{array}$ & $p=0.009 *$ \\
\hline Median age of menopause, years (IQR); min-max $(n=23)$ & $\begin{array}{l}54.00(2.00) \\
49.00-55.00\end{array}$ & $\begin{array}{l}52.00(4.00) \\
48.00-55.00\end{array}$ & $\begin{array}{l}52.00(1.00) \\
50.00-54.00\end{array}$ & $p=0.061$ \\
\hline Median duration of menstrual cycle, days (IQR); min-max & $\begin{array}{l}28.00(2.00) \\
24.00-32.00\end{array}$ & $\begin{array}{l}28.00(1.00) \\
25.00-34.00\end{array}$ & $\begin{array}{l}28.00(0.00) \\
26.00-31.00\end{array}$ & $p=0.408$ \\
\hline Median duration of menstruation, days (IQR), min-max & $\begin{array}{l}4.00(1.00) \\
3.00-7.00\end{array}$ & $\begin{array}{l}4.00(1.00) \\
3.00-7.00\end{array}$ & $\begin{array}{l}4.00(1.00) \\
3.00-6.00\end{array}$ & $p=0.281$ \\
\hline $\begin{array}{l}\text { Menstrual cycles, } \mathrm{n}(\%) \\
\text { Regular } \\
\text { Irregular }\end{array}$ & $\begin{array}{l}36(67.92 \%) \\
17(32.08 \%)\end{array}$ & $\begin{array}{l}32(60.38 \%) \\
21(39.62 \%)\end{array}$ & $\begin{array}{l}42(79.25 \%) \\
\text { II (20.75\%) }\end{array}$ & $p=0.164$ \\
\hline $\begin{array}{l}\text { Menstrual cycles, n (\%) } \\
\text { Painful } \\
\text { Painless }\end{array}$ & $\begin{array}{l}13(24.53 \%) \\
40(75.47 \%)\end{array}$ & $\begin{array}{l}15(28.31 \%) \\
38(71.69 \%)\end{array}$ & $\begin{array}{l}9(16.98 \%) \\
44(83.08 \%)\end{array}$ & $p=0.510$ \\
\hline $\begin{array}{l}\text { Type of menstrual bleeding, n (\%) } \\
\text { Scant } \\
\text { Normal } \\
\text { Heavy }\end{array}$ & $\begin{array}{l}4(7.55 \%) \\
37(69.81 \%) \\
12(22.64 \%)\end{array}$ & $\begin{array}{l}2(3.77 \%) \\
36(67.92 \%) \\
15(28.31 \%)\end{array}$ & $\begin{array}{l}4(7.55 \%) \\
46(86.79 \%) \\
3(5.66 \%)\end{array}$ & $p=0.038 *$ \\
\hline $\begin{array}{l}\text { Parity, n (\%) } \\
\text { Nullipara } \\
\text { Primipara } \\
\text { Multipara }\end{array}$ & $\begin{array}{l}5(9.43 \%) \\
4(7.55 \%) \\
44(83.02 \%)\end{array}$ & $\begin{array}{l}3(5.66 \%) \\
9(16.98 \%) \\
4 I(77.36 \%)\end{array}$ & $\begin{array}{l}2(3.77 \%) \\
5(9.43 \%) \\
46(86.80 \%)\end{array}$ & $p=0.403$ \\
\hline
\end{tabular}

Abbreviations: FIGO, International Federation of Gynecology and Obstetrics classification of uLMS; IQR, interquartile range.

were noticed, except for the type of menstrual bleeding between donors of uM, uLM, and uLMS specimens; women with uLMS and uLM reported statistically significantly more frequent abnormal vaginal spotting than controls $(21 / 53$ [39.62\%] vs 6/53 [11.32\%], $p<0.001$, and $26 / 53$ [49.06\%] vs $6 / 53$ [11.32\%], $p<0.001$, respectively; Table 1).

\section{DFF40, DFF45, and Bcl-2 expression in uterine leiomyosarcomas}

Bcl-2 showed cytoplasmic localization, while both DFF40 and DFF45 showed a nuclear pattern of expression (Figure 1). Only seven (13.21\%) uLMS tissues had moderate expression of DFF40 and three (5.66\%) showed moderate expression 


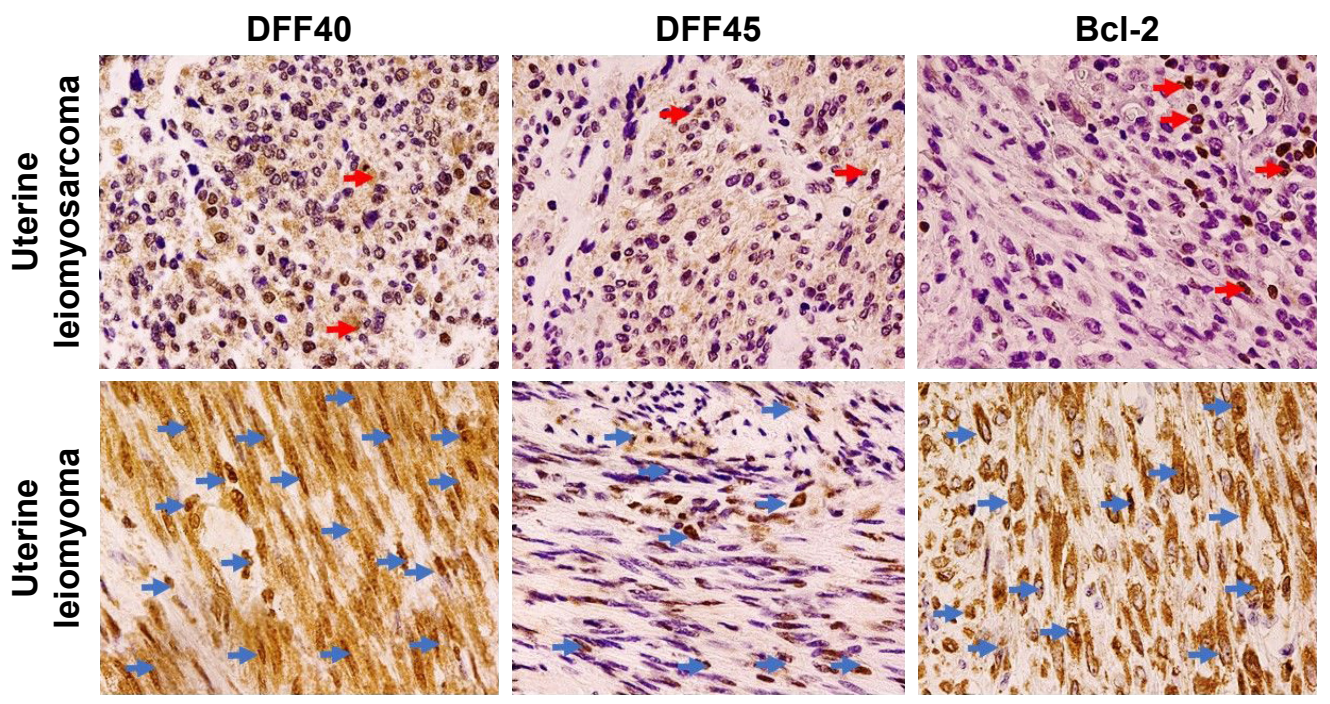

Figure I Weak DFF40, DFF45, and Bcl-2 expression (400x) in uterine leiomyosarcomas (red arrows) compared to strong DFF40, Bcl-2, and moderate DFF45 expression in uterine leiomyomas (blue arrows).

Abbreviations: DFF40, DNA fragmentation factor 40; DFF45, DNA fragmentation factor 45; Bcl-2, B-cell lymphoma 2 protein.

of Bcl-2. High expression of the studied proteins was absent from all uLMS samples (Table 2). DFF40 expression in uLMS was significantly lower than that in both uM $(p<0.001)$ and uLMS-M $(p<0.001)$. Similarly, DFF45 expression in uLMS was significantly lower than that in $\mathrm{uM}(p<0.001)$ and uLMS-M $(p<0.001$; Table 2$)$. Bcl-2 expression in uLMS specimens was also significantly lower than that in $\mathrm{uM}(p<0.001)$ and uLMS-M $(p=0.001$; Table 2$)$. In uLMS specimens, no correlations between DFF40 and DFF45, Bcl-2 and DFF40, or Bcl-2 and DFF45 expression were observed. Bcl-2 showed a significant inverse moderate correlation with tumor stage $(\mathrm{R}=-0.368 ; p=0.007)$ and MI ( $\mathrm{R}=-0.301 ; p=0.029)$, but not with tumor diameter or grade. DFF40 but not DFF45 correlated inversely with the MI ( $\mathrm{R}=-328 ; p=0.016$ ), whereas both DFF40 and DFF45 correlated negatively with the stage of the disease $(\mathrm{R}=-0.300$; $p=0.029 ; \mathrm{R}=-0.301 ; p=0.029$, respectively); however, their expression did not correlate with tumor grade.

Women with uLMS that was negative for DFF40 and Bcl-2 staining showed significantly shorter OS than the OS of those whose specimens showed positive staining $(p<0.001$ and $p<0.001$, respectively), while there was no relationship between DFF45 expression and OS (Figure 2). OS in patients with stages III and IV disease $(p<0.001), \mathrm{a} \mathrm{G}_{3}$ tumor $(p=0.016)$, and an MI over 50 was poorer (Figure 2). Similarly, patients whose specimens tested negative for DFF40 and Bcl-2 (but not DFF45; $p=0.342$ ) expression showed significantly shorter DFS $(p<0.001$ and $p<0.001$, respectively; Figure 3 ). Patients with advanced stages of disease and higher grades and MI also had poorer DFS $(p<0.001$, $p=0.028$, and $p<0.001$, respectively; Figure 2).
Negative DFF40 and Bcl-2 expression, but not DFF45, were adverse prognostic factors for DFS and OS in women with uLMS. Further analysis showed that an advanced disease stage, high tumor grade, and elevated MI were associated with a reduction in OS and DFS (Table 3).

\section{DFF40, DFF45, and Bcl-2 expression in uLM}

uLM showed marked nuclear expression of DFF40/DFF45 and cytoplasmic expression of Bcl-2 (Figure 2). DFF40 expression in $\mathrm{ULM}$ was significantly higher than that in $\mathrm{uM}$ and uLM-M ( $p=0.001$ and $p=0.005$, respectively; Table 2 ). No significant differences in DFF45 scoring between uLM and $\mathrm{uM}$, nor between $\mathrm{uLM}$ and $\mathrm{uLM}-\mathrm{M}$ were observed (Table 2). In addition, uLM showed the highest Bcl-2 expression, as compared with uM $(p<0.001)$ and uLM-M $(p<0.001)$. In $u L M$, we found a significant positive correlation between DFF40 and DFF45 expression ( $\mathrm{R}=0.388$; $p=0.004)$ and between DFF 40 and Bcl- 2 expression $(\mathrm{R}=0.630$; $p<0.001)$; however, there was no correlation between DFF45 and Bcl-2 expression. Bcl-2 expression correlated positively and significantly with the diameter of the leiomyoma $(\mathrm{R}=0.435 ; p<0.001)$.

\section{DFF40, DFF45, and Bcl-2 expression in uterine myometrium}

Myometrial samples showed a nuclear pattern of DFF40/ DFF45 and cytoplasmic of Bcl-2 staining. Only four (7.55\%) samples of the control uM were DFF40-negative, whereas 30 $(56.60 \%)$ presented with low, $16(30.19 \%)$ with moderate, and three (5.66\%) with high DFF40 expression. Similarly, 


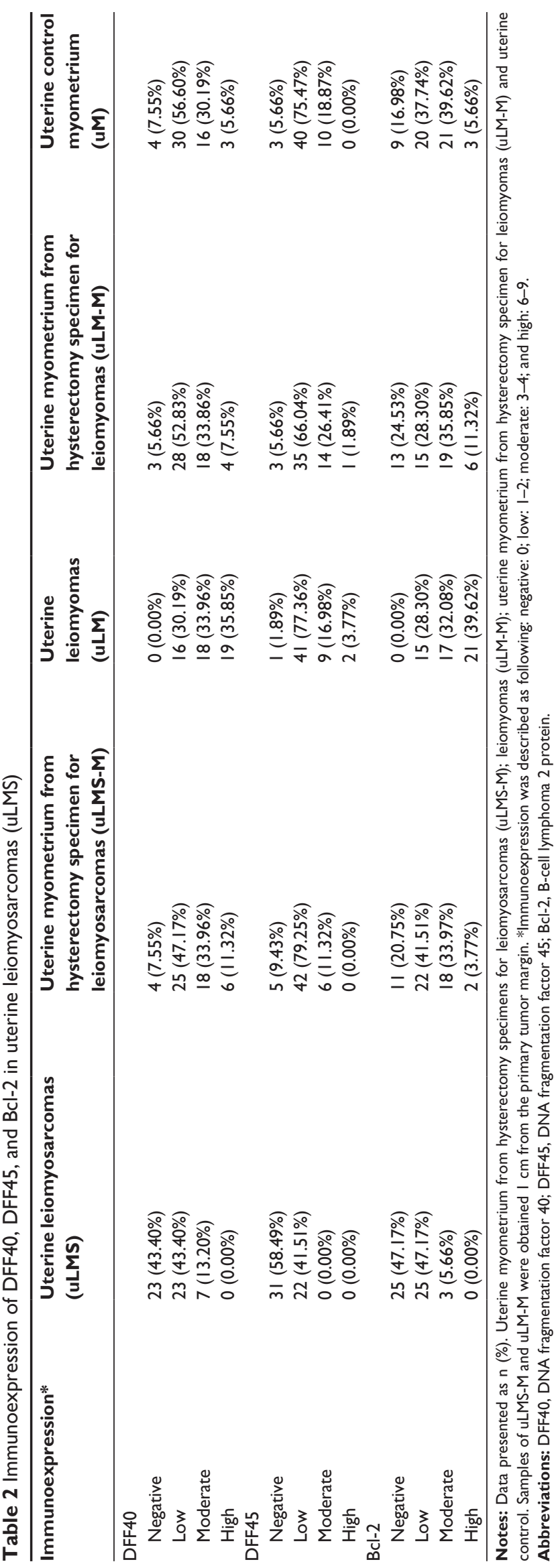

uLMS-M and uLM-M showed predominately low and moderate DFF40 expression and scantly low and high expression (Table 2). No significant differences were observed in DFF40 nor DFF45 scoring between uM, uLMS-M, and uLM-M (Table 2). Bcl-2 showed mainly moderate expression in $\mathrm{uM}$ and uLM-M, and low in uLMS-M (Table 2). No significant differences were observed in Bcl-2 expression between the uM, uLM-M, and uLMS-M groups.

In $\mathrm{uM}$, a moderate positive and significant correlation between DFF40 and DFF45 expression was observed $(\mathrm{R}=0.762 ; p<0.001)$, with no correlation between Bcl-2 and DFF40 or Bcl-2 and DFF45. In uLM-M, a strong positive and significant correlation between DFF40 and DFF45 was also proven $(\mathrm{R}=0.893 ; p<0.001)$, with no association observed between Bcl-2 and DFF40 or Bcl-2 and DFF45. Additionally, in uLMS-M specimens, the only correlation observed was that between DFF40 and DFF45 $(\mathrm{R}=0.703$; $p<0.001)$.

\section{Validation of the intra-rater reliability for immunohistochemistry scoring}

An excellent intra-rater agreement was confirmed with regard to the immunoscoring of DFF40, DFF45, and Bcl-2 expression, and the following values were noted:

A) The first pathologist (ie, observer 1 vs 1) for the assessment of DFF40 showed, ICC 0.976 [confidence interval (CI) 0.959-0.986]; for DFF45, ICC 0.934 (CI 0.900-0.961); and for Bcl-2, ICC 0.952 (CI 0.919-0.972).

B) The second pathologist (ie, observer 2 vs 2) for the assessment of DFF40, ICC 0.934 (CI 0.889-0.961); for DFF45, ICC 0.898 (CI 0.831-0.929); and for Bcl-2, ICC 0.984 (CI 0.972-0.990).

\section{Discussion}

To our knowledge, a comprehensive investigation of DFF40 and DFF45 immunoexpression along with Bcl-2 staining in leiomyosarcomas and leiomyomas of the human uterus has not been reported previously. Many studies have confirmed Bcl-2 underexpression in uLMS and have identified it as a predictor of poor OS; in contrast, uLM shows increased expression of this protein. ${ }^{17,32,41-43}$ Our outcomes confirmed that the highest Bcl-2 expression was present in uLM, and the lowest in uLMS. Similarly, Bcl-2-negative uLMS cases presented with a significantly shorter DFS and OS. Additionally, uLM specimens showed a lack of correlation between Bcl-2 and DFF40 or DFF45 expression.

As we found no studies concerning DFF40/DFF45 expression in uLMS, our findings can only be discussed in the context of other malignancies. Both DFF40 and DFF45 

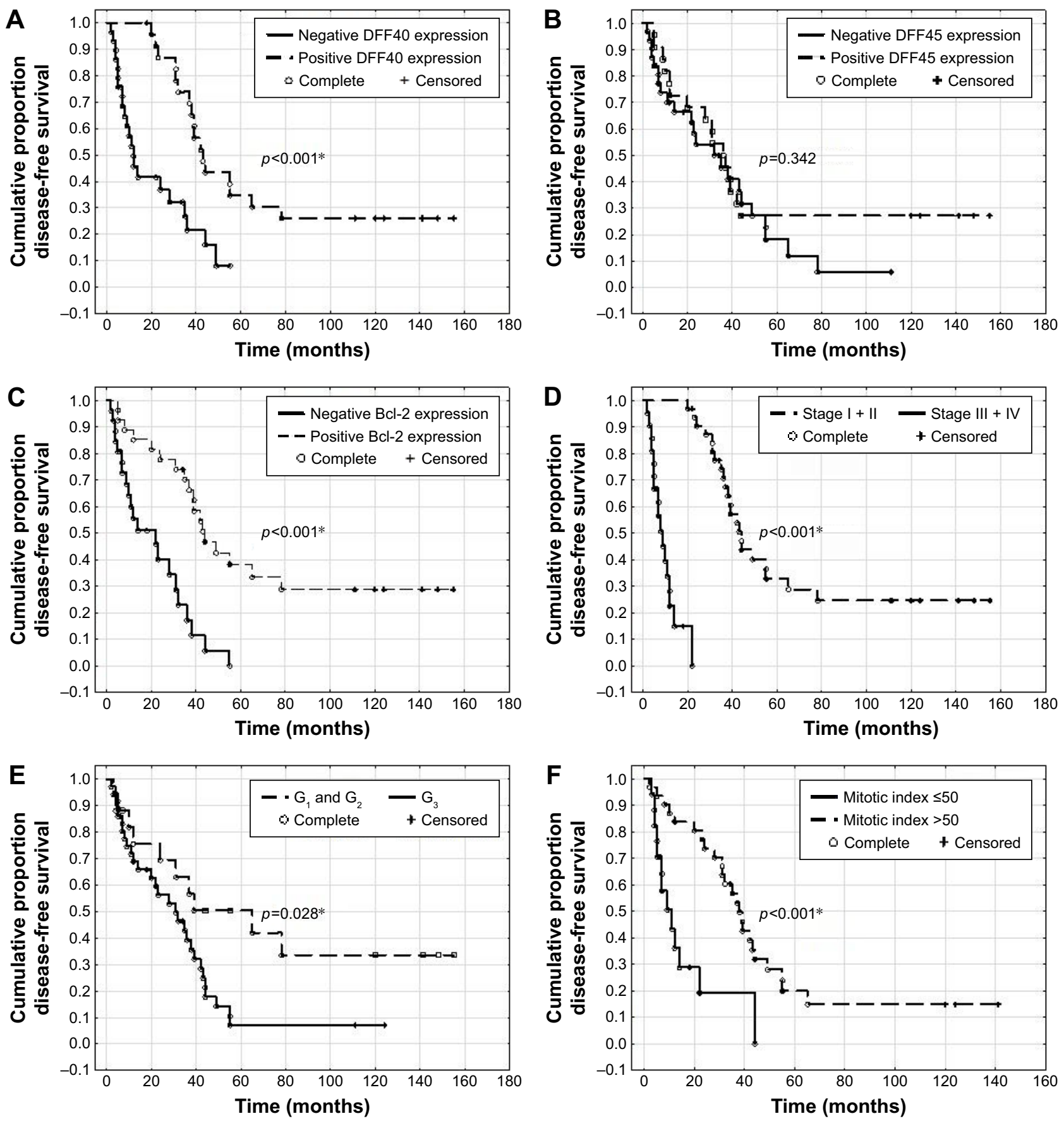

Figure 2 Disease-free survival (DFS) in women with leiomyosarcomas depending on DFF40 (A), DFF45 (B), and Bcl-2 (C) expression and disease stage (D) according to FIGO classification, tumor grade $(\mathbf{E})$, and mitotic index $(\mathbf{F}) ;{ }^{*}<0.05$ is statistically significant.

Abbreviations: DFF40, DNA fragmentation factor 40; DFF45, DNA fragmentation factor 45; Bcl-2, B-cell lymphoma 2 protein; FIGO, International Federation of Gynecology and Obstetrics.

were underexpressed in uLMS, and DFF40-negative cases presented with significantly shorter DFS and OS; in contrast, DFF45 expression was not a predictive factor of OS or DFS. Sánchez-Osuna et al reported that DFF40-deficient highgrade glioblastomas showed incomplete apoptosis, despite the correct activation of executioner caspases. ${ }^{44}$ Bagheri et al reported that DFF40 overexpression sensitized breast cancer cells to doxorubicin, concluding that modulation of DFF40 levels may be a beneficial strategy for treatment of chemoresistant cancers. ${ }^{45}$ Additionally, Boon and Sim reported that the overexpression of only DFF40 in nasopharyngeal carcinomas did not result in increased apoptosis of head and neck squamous carcinoma in response to oxidative stress, as DFF45 was required for proper DFF40 folding. ${ }^{46}$

DFF45 deficiency was also reported as a cause of abnormal cell apoptosis; this seems counterintuitive, as DFF45 is an 

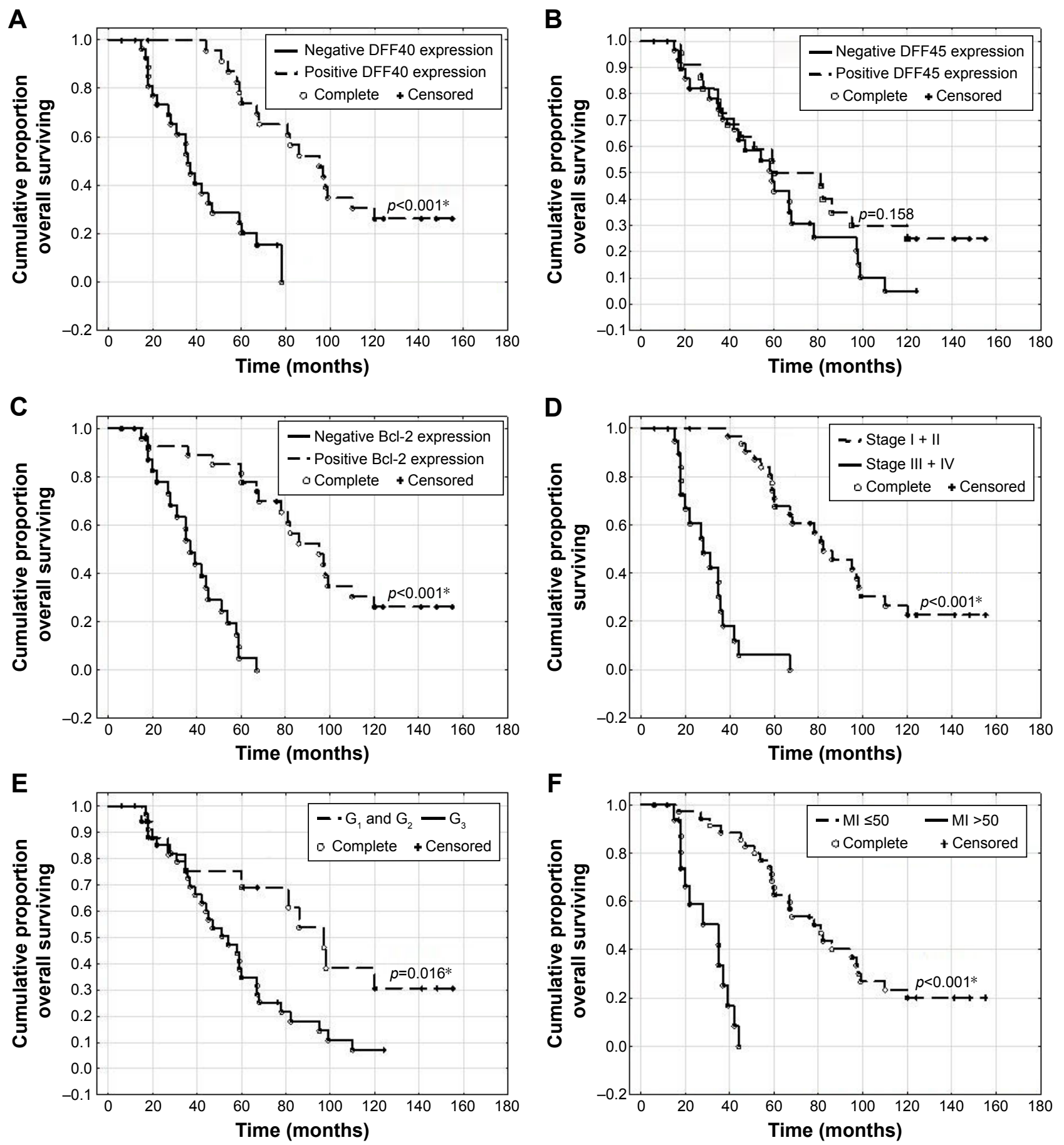

Figure 3 Overall survival (OS) in women with leiomyosarcomas depending on DFF40 (A), DFF45 (B), and Bcl-2 (C) expression, and disease stage (D) according to FIGO classification, tumor grade (E), and mitotic index $(\mathbf{F}) ;{ }^{*} p<0.05$ is statistically significant.

Abbreviations: DFF40, DNA fragmentation factor 40; DFF45, DNA fragmentation factor 45; Bcl-2, B-cell lymphoma 2 protein; FIGO, International Federation of Gynecology and Obstetrics.

inhibitor of DFF40 and, thus, the lack of an inhibitor should result in increased activity of DNAse. However, it is important to note that DFF45 also acts as a chaperone for DFF40 and its presence is mandatory for proper DFF40 folding, which is, in turn, essential to the functional activation of DFF40. Errami et al proved that DFF45-negative colon cancer cells were more resistant to apoptosis than was normal colon tissue. ${ }^{35}$
Furthermore, Konishi et al showed that esophageal squamous cancer cells exhibit abundant DFF45 expression, which correlated negatively with tumor grade and enhanced lymphatic spread. ${ }^{34}$ The only study to investigate DFF45 expression in gynecological malignancies, conducted by Brustmann, showed that DFF45 upregulation in serous ovarian cancer was correlated with aggressive tumor behavior. ${ }^{47}$ Another study 
Table 3 Cox's proportional hazard regression analysis of the prognostic value of clinical and immunohistochemical variables in 53 women with uterine leiomyosarcomas

\begin{tabular}{|c|c|c|c|c|}
\hline \multirow[t]{2}{*}{ Prognostic factor } & \multicolumn{2}{|c|}{ Disease free survival (DFS) } & \multicolumn{2}{|l|}{ Overall survival (OS) } \\
\hline & HR (CI) & $P$-value & HR (CI) & $p$-value \\
\hline Age & $0.973(0.92 I-I .029)$ & 0.348 & $0.964(0.910-1.020)$ & 0.206 \\
\hline Stage 3+ & $9.024(1.196-81.968)$ & $<0.00 \mathrm{I}^{\#}$ & I4.153 (6.06|-33.049) & $<0.00 I^{\#}$ \\
\hline Grade 3 & $2.226(1.064-4.656)$ & $0.031^{\#}$ & $2.396(1.148-4.999)$ & $0.019^{\#}$ \\
\hline $\mathrm{MI}>50$ & $4.674(2.184-10.014)$ & $<0.00 I^{\#}$ & I8.60| (5.703-60.670) & $<0.00 I^{\#}$ \\
\hline Negative DFF40 staining & $3.254(3.254-6.459)$ & $0.007^{\#}$ & $6.315(2.7 \mid 2-14.704)$ & $<0.00 I^{\#}$ \\
\hline Negative DFF45 staining & I.359 (0.7I5-2.583) & 0.349 & $1.587(0.829-3.044)$ & 0.164 \\
\hline Negative Bcl-2 staining & $3.893(1.940-7.812)$ & $<0.00 I^{\#}$ & I I.476 (4.454-29.564) & $<0.00 I^{\#}$ \\
\hline
\end{tabular}

Note: ${ }^{*} p$, significant $p$ value.

Abbreviations: DFF40, DNA fragmentation factor 40; DFF45, DNA fragmentation factor 45; Bcl-2, B-cell lymphoma 2 protein; Cl, confidence intervals; HR, hazard ratio; MI, mitotic index

also showed increasing DFF45 immunoreactivity from normal endometrium through normal endometrial and atypical hyperplasia to endometrial adenocarcinomas. ${ }^{48}$ The results of DFF45 expression in different malignancies are inconclusive. In our study, we observed altered DFF45 staining in uLMS that correlated only with the stage of the disease, but did not influence DFS or OS.

Apoptosis is mainly triggered by either membrane death receptors or via the mitochondrial pathway, involving activation of many proapoptotic proteins and deactivation of anti-apoptotic factors. ${ }^{49,50}$ Furthermore, under persistent exposure to damaging agents, the endoplasmatic reticulum can initiate apoptosis through calcium release and activation of caspase $12 .{ }^{51}$ These mechanisms activate a cascade of caspases that exist mainly in the cytosol as pro-enzymes; a cross-talk between apoptotic factors then finally results in caspase-3 activation that cleaves DFF45, releasing DFF40, which is directly responsible for the last step of apoptosis: DNA laddering. ${ }^{24}$ Increased Bcl-2 expression in leiomyomas may explain an uncontrolled tumor proliferation, and confirms the monoclonal hypothesis of uterine fibroid formation. ${ }^{9}$ In contrast to high Bcl-2 expression, we would rather have expected decreased DFF40 levels in uLM, as this protein results in the DNA damage. In uLMS, low levels of DFF40 were intuitively predicted, although we expected higher levels of Bcl-2 as an anti-apoptotic protein. These apparently inconsistent and counterintuitive results are in agreement with other reports showing increased expression of some oncogenes in benign proliferative disorders and their depletion in malignancies. ${ }^{27}$

Based on these findings, we hypothesize that benign tumors and malignancies may have developed different mechanisms to avoid apoptosis. In uLM, Bcl-2 overexpression seems important for preventing cells from undergoing apoptosis. High DFF40 levels make leiomyoma cells more susceptible to apoptosis; however, the proapoptotic signal seems to be abrogated at earlier levels of the apoptotic cascade, before activation of DFF40/DFF45, and Bcl-2 overexpression can enhance these mechanisms. Second, an exclusive increase in DFF40 expression, without a DFF45 increase, may be not sufficient for proper DFF40 activation, as the presence of DFF45 is essential for DFF40 to acquire its biological function. Unbalanced DFF40/ DF45 expression may be another mechanism allowing leiomyoma cells to escape apoptosis. In uLMS, significant decreases in DFF40 and DFF45 expression seem sufficient to prevent DNA laddering. Under these circumstances, functioning of factors involved in transmitting of pro- and anti-apoptotic signals (including low Bcl-2 expression) seems secondary.

In this study, we confirmed that DFF40, DFF45, and Bcl-2 underexpression in uLMS can enhance apoptosis dysregulation. Moreover, in malignant tissues, no correlation was found between DFF40 and DFF45 expression. Dysregulation of DFF40 and DFF45 expression may be another mechanism underlying cell escape from apoptosis, as the presence of DFF45 is mandatory for proper DFF40 function.

The main strength of the study is that it provides a comprehensive analysis of DFF40 and DFF45, simultaneously with Bcl-2 evaluation, in uLMS. We correlated DFF40 and DFF45 expression with clinical malignancies and pathological features, showing that DFF40 and Bcl-2, but not DFF45, may serve as independent prognostic factors of DFS and OS. Moreover, protein expression in uLMS was not only compared with their expression in normal human myometrium, but also with cases of leiomyomas and myometrium from corresponding cases. In this analysis, no differences between case and control myometrium were seen in DFF40, DFF45, and Bcl-2 expression; these observations agree with the clonal theory of uLMS and uLM, which confirms the very low risk of malignant transformation of uLM. 
Finally, we observed a positive correlation between DFF40 and DFF45 expression but not Bcl-2, in both control and case myometrial samples.

The use of immunohistochemistry, which is a semiquantitative method, may be considered a potential limitation of the study, especially for comparing DFF40 and DFF45 expression in the same tissue. We acknowledge that an immunostaining analysis is subjective and, for that reason, each sample was evaluated by two highly experienced pathologists. This methodology is widely accepted and has been employed by many other studies for investigating the expression of DFF45 and Bcl-2. ${ }^{25-29,32}$ As complete inter-rater agreement was achieved, the intra-rater disparity was the only potential bias in our sample assessment. However, as this disparity showed an almost-perfect correlation, intra-rater bias can safely be excluded.

\section{Conclusion}

This current study provides important evidence regarding the underexpression of DFF40/DFF45 and Bcl-2 in uLMS, and shows that DFF40 and Bcl-2 may serve as prognostic factors for DFS and OS. This comprehensive evaluation provides more insight into other findings concerning DFF40, DFF45, and $\mathrm{Bcl}-2$ expression in malignancies.

\section{Acknowledgments}

The authors thank Dr H Molak-Olczak, who independently and blindly analyzed the immunoexpression of the investigated proteins as a second specialist in histopathology.

\section{Author contributions}

TB was the chief investigator who designed the study, selected the cases, conducted data analysis, and drafted the manuscript. KP and KO participated in the creation of the study design, specimen evaluation, and the selection of eligible cases. $\mathrm{KO}$ performed the protein immunoexpression assessment. AC performed statistical analysis and critically reviewed the manuscript. All authors contributed toward data analysis, drafting and critically revising the paper and agree to be accountable for all aspects of the work.

\section{Disclosure}

The authors report no conflicts of interest in this work.

\section{References}

1. D'Angelo E, Prat J. Uterine sarcomas: a review. Gynecol Oncol. 2010; 116(1):131-139.

2. Zhiqiang L, Bin S, Min F, Yufang L. Leiomyosarcoma of cervical stump following subtotal hysterectomy: a case report and review of literature. Eur J Gynaecol Oncol. 2016;37(1):148-151.
3. Khosla D, Gupta R, Srinivasan R, Patel FD, Rajwanshi A. Sarcomas of uterine cervix: clinicopathological features, treatment, and outcome. Int J Gynecol Cancer. 2012;22(6):1026-1030.

4. Boll D, Verhoeven RH, van der Aa MA, et al. Incidence and survival trends of uncommon corpus uteri malignancies in the Netherlands, 1989-2008. Int J Gynecol Cancer. 2012;22(4):599-606.

5. Amant F, Coosemans A, Debiec-Rychter M, Timmerman D, Vergote I. Clinical management of uterine sarcomas. Lancet Oncol. 2009;10(12): 1188-1198.

6. Gottlieb S. Tamoxifen may increase risk for uterine sarcoma. $\mathrm{Br}$ Med J. 2002;325:7.

7. Naaman Y, Shveiky D, Ben-Shachar I, Shushan A, Mejia-Gomez J, Benshushan A. Uterine sarcoma: prognostic factor and treatment evaluation. Isr Med Assoc J. 2011;13(2):76-79.

8. NCCN Clinical Practice Guidelines in Oncology. Uterine Neoplasms. Version 2. 2016. Accessed from: www.nccn.org. Accessed December 7, 2016.

9. Cramer SF, Patel A. The frequency of uterine leiomyomas. Am J Clin Pathol. 1990;94(4):435-438.

10. Mittal K, Joutovsky A. Areas with benign morphologic and immunohistochemical features are associated with some uterine leiomyosarcomas. Gynecol Oncol. 2007;104(2):362-365.

11. Mittal K. Precursor lesions for uterine leiomyosarcoma. Hum Pathol. 2007;38(8):1289.

12. Zhang $\mathrm{P}$, Zhang $\mathrm{C}$, Hao J, et al. Use of X-chromosome inactivation pattern to determine the clonal origins of uterine leiomyoma and leiomyosarcoma. Hum Pathol. 2006;37(10):1350-1356.

13. Gadducci A, Sartori E, Landoni F, et al. The prognostic relevance of histological type in uterine sarcomas: a Cooperation Task Force (CTF) multivariate analysis of 249 cases. Eur J Gynaecol Oncol. 2002; 23(4):295-289.

14. Raspollini MR, Amunni G, Villanucci A, et al. Estrogen and progesterone receptors expression in uterine malignant smooth muscle tumors: correlation with clinical outcome. J Chemother. 2003;15(6):596-602.

15. Smirnova IS, Yakovleva TK, Rosanov YM, Aksenov ND, Pospelova TV. Analysis of p53-dependent mechanisms in the maintenance of genetic stability in diploid tumourigenic line SK-UT-1B of human uterine leiomyosarcoma. Cell Biol Int. 2001;25(11):1101-1115.

16. Kułak K, Bobiński M, Polak G, Jedrych BJ, Kotarski J, Bednarek W. Ocena ekspresji kaspazy 9 i bialka p53 w miesakach macicy. [Expression of caspase 9 and p53 in uterine leiomyosarcomas]. Ginekol Pol. 2014;85(8):600-604. Polish

17. Bodner K, Bodner-Adler B, Kimberger O, Czerwenka K, Mayerhofer K. Bcl-2 expression and other clinicopathologic parameters in uterine leiomyosarcoma. Wien Klin Wochenschr. 2004;116(4):135-139.

18. Kowalewska M, Bakula-Zalewska E, Chechlinska M, et al. microRNAs in uterine sarcomas and mixed epithelial-mesenchymal uterine tumors: a preliminary report. Tumour Biol. 2013;34(4):2153-2160.

19. Barlin JN, Zhou QC, Leitao MM, et al. Molecular subtypes of uterine leiomyosarcoma and correlation with clinical outcome. Neoplasia. 2015;17(2):183-189.

20. Cekanova M, Fernando RI, Siriwardhana N, et al. BCL-2 family protein, $\mathrm{BAD}$ is down-regulated in breast cancer and inhibits cell invasion. Exp Cell Res. 2015;331(1):1-10.

21. Chaudhry P, Srinivasan R, Patel FD. Differential expression of Fas family members and Bcl-2 family members in benign versus malignant epithelial ovarian cancer (EOC) in North Indian population. Mol Cell Biochem. 2012;368(1-2):119-126.

22. Dorjgochoo T, Xiang YB, Long J, et al. Association of genetic markers in the BCL-2 family of apoptosis-related genes with endometrial cancer risk in a Chinese population. PLoS One. 2013;8(4):e60915.

23. Mao PL, Jiang Y, Wee BY, Porter AG. Activation of caspase-1 in the nucleus requires nuclear translocation of pro-caspase-1 mediated by its prodomain. J Biol Chem. 1998;273(37):23621-23624.

24. Fukushima K, Kikuchi J, Koshiba S, Kigawa T, Kuroda Y, Yokoyama S. Solution structure of the DFF-C domain of DFF45/ICAD. A structural basis for the regulation of apoptotic DNA fragmentation. $J$ Mol Biol. 2002;321(2):317-327. 
25. Schorr K, Li M, Krajewski S, Reed JC, Furth PA. Bcl-2 gene family and related proteins in mammary gland involution and breast cancer. J Mammary Gland Biol Neoplasia. 1999;4(2):153-164.

26. Khodapasand E, Jafarzadeh N, Farrokhi F, Kamalidehghan B, Houshmand $\mathrm{M}$. Is Bax/Bcl-2 ratio considered as a prognostic marker with age and tumor location in colorectal cancer? Iran Biomed J. 2015; 19(2):69-75.

27. Nakamura T, Nomura S, Sakai T, Nariya S. Expression of bcl-2 oncoprotein in gastrointestinal and uterine carcsinomas and their premalignant lesions. Hum Pathol. 1997;28(3):309-315.

28. Geisler JP, Geisler HE, Wiemann MC, Zhou Z, Miller GA, Crabtree W. Lack of bcl-2 persistence: an independent prognostic indicator of poor prognosis in endometrial carcinoma. Gynecol Oncol. 1998;71(2): 305-307.

29. Hwang KT, Han W, Kim J, et al. Prognostic influence of BCL2 on molecular subtypes of breast cancer. J Breast Cancer. 2017;20(1):54-64.

30. Porichi O, Nikolaidou ME, Apostolaki A, et al. BCL-2, BAX and P53 expression profiles in endometrial carcinoma as studied by realtime PCR and immunohistochemistry. Anticancer Res. 2009;29(10): 3977-3982.

31. Geisler JP, Geisler HE, Wiemann MC, Zhou Z, Miller GA, Crabtree W. Lack of bcl-2 persistence: an independent prognostic indicator of poor prognosis in endometrial carcinoma. Gynecol Oncol. 1998;71(2): 305-307

32. Zhai YL, Nikaido T, Toki T, Shiozawa A, Orii A, Fujii S. Prognostic significance of bcl-2 expression in leiomyosarcoma of the uterus. Br J Cancer. 1999;80(10):1658-1664.

33. Rajandram R, Razack AH, Ng KL, Gobe GC. Decreased expression of inhibitor of caspase-activated DNase (ICAD) in renal cell carcinoma - tissue microarray of human samples. J Kidney Cancer VHL. 2016;3(1):1-11.

34. Konishi S, Ishiguro H, Shibata Y, et al. Decreased expression of DFF45/ ICAD is correlated with a poor prognosis in patients with esophageal carcinoma. Cancer. 2002;95(12):2473-2478.

35. Errami Y, Brim H, Oumouna-Benachour K, et al. ICAD deficiency in human colon cancer and predisposition to colon tumorigenesis: linkage to apoptosis resistance and genomic instability. PLoS One. 2013; 8(2):e57871.

36. Banas T, Basta P, Knafel A, et al. DFF45 expression in human endometrium is associated with menstrual cycle phases and decreases after menopause. Gynecol Obstet Invest. 2012;73:177-182.

37. Banas T, Skotniczny K, Basta A. DFF45 expression in ovarian endometriomas. Eur J Obstet Gynecol Reprod Biol. 2009;146(1):87-91.
38. Remmele W, Stegner HE. Recommendation for uniform definition of an immunoreactive score (IRS) for immunohistochemical estrogen receptor detection (ER-ICA) in breast cancer tissue. Pathologe. 1987;8(3): 138-140. [Article in German]

39. Preacher KJ. Calculation for the chi-square test: an interactive calculation tool for chi-square tests of goodness of fit and independence [Computer software]. Available from: http://quantpsy.org. Accessed January 2, 2017.

40. Kottner J, Audige L, Brorson S, et al. Guidelines for reporting reliability and agreement studies (GRRAS) were proposed. Int J Nurs Stud. 2011 48(6):661-671.

41. Kobayashi H, Uekuri C, Akasaka J, et al. The biology of uterine sarcomas: a review and update. Mol Clin Oncol. 2013;1(4):599-609.

42. Leiser AL, Anderson SE, Nonaka D, et al. Apoptotic and cell cycle regulatory markers in uterine leiomyosarcoma. Gynecol Oncol. 2006; 101(1):86-91.

43. D'Angelo E, Espinosa I, Ali R, et al. Uterine leiomyosarcomas: tumor size, mitotic index, and biomarkers $\mathrm{Ki} 67$, and Bcl-2 identify two groups with different prognosis. Gynecol Oncol. 2011;121(2):328-333.

44. Sánchez-Osuna M, Martínez-Escardó L, Granados-Colomina C, et al. An intrinsic DFF40/CAD endonuclease deficiency impairs oligonucleosomal DNA hydrolysis during caspase-dependent cell death: a common trait in human glioblastoma cells. Neuro Oncol. 2016;18(7): 950-961.

45. Bagheri F, Safarian S, Eslaminejad MB, Sheibani N. Sensitization of breast cancer cells to doxorubicin via stable cell line generation and overexpression of DFF40. Biochem Cell Biol. 2015;93(6):604-610.

46. Boon SS, Sim SP. Inhibitor of caspase-activated DNase expression enhances caspase-activated DNase expression and inhibits oxidative stress-induced chromosome breaks at the mixed lineage leukaemia gene in nasopharyngeal carcinoma cells. Cancer Cell Int. 2015;15:54.

47. Brustmann H. DNA fragmentation factor (DFF45): expression and prognostic value in serous ovarian cancer. Pathol Res Pract. 2006; 202(10):713-720.

48. Brustmann H. Poly(ADP-ribose) polymerase (PARP) and DNAfragmentation factor (DFF45): expression and correlation in normal, hyperplastic and neoplastic endometrial tissues. Pathol Res Pract. 2007; 203(2):65-72.

49. Nagata S. Apoptosis by death factor. Cell. 1997;88(3):355-356.

50. Leist M, Jäättelä M. Four deaths and a funeral: from caspases to alternative mechanisms. Nat Rev Mol Cell Biol. 2001;2(8):589-598.

51. Berridge MJ, Lipp P, Bootman MD. The versatility and universality of calcium signaling. Nat Rev Mol Cell Biol. 2000;1(1):11-21.
OncoTargets and Therapy

\section{Publish your work in this journal}

OncoTargets and Therapy is an international, peer-reviewed, open access journal focusing on the pathological basis of all cancers, potential targets for therapy and treatment protocols employed to improve the management of cancer patients. The journal also focuses on the impact of management programs and new therapeutic agents and protocols on

\section{Dovepress}

patient perspectives such as quality of life, adherence and satisfaction. The manuscript management system is completely online and includes a very quick and fair peer-review system, which is all easy to use. Visit http://www.dovepress.com/testimonials.php to read real quotes from published authors. 